\title{
Synthesis and Self-Assembly of Functionalized
}

\section{Calix[8]arenes on Graphite}

Jian Zhang, ${ }^{\dagger}$ Ganna Podoprygorina, ${ }^{\ddagger}$ Vasiliy Brusko, ${ }^{*, \S}$ Volker Böhmer, ${ }^{*, *}$ and Andreas Janshoff ${ }^{*, \dagger}$

Institut für Physikalische Chemie, Fachbereich Chemie und Pharmazie, Johannes Gutenberg-

Universität, Welderweg 11, D-55099 Mainz, Germany and Abteilung Lehramt Chemie, Fachbereich

Chemie und Pharmazie, Johannes Gutenberg-Universität, Duesbergweg 10-14, D-55099 Mainz, Germany

\section{Supporting Information}

Synthesis of 3,6-didecyloxy-1,8-naphthalic anhydride. $\mathrm{NaH}(0.062 \mathrm{~g}, 2.564 \mathrm{mmol})$ was added to a solution of 3,6-dihydroxy-1,8-naphthalic anhydride $(0.281 \mathrm{~g}, 1.221 \mathrm{mmol})$ in DMF (40 ml) at $\mathrm{rt}$ under nitrogen. The dark-red reaction mixture was stirred for $20 \mathrm{~min}$ before decyl iodide $(0.91 \mathrm{ml}, 3.662$ mmol) was added. After $12 \mathrm{~h}$ at $\mathrm{rt}$ a beige precipitate had formed. Then glacial acetic acid $(20 \mathrm{~mL})$ followed by cold water $(100 \mathrm{ml})$ were poured carefully to the stirred mixture. The solid was filtered off, washed with water and methanol. Recrystallization from acetone gave the desired anhydride as white powder. Yield: $0.623 \mathrm{~g}(72 \%)$; mp $162^{\circ} \mathrm{C}$; MS (FD): m/z $402.5\left(\mathrm{M}^{+}\right) ;{ }^{1} \mathrm{H}$ NMR $\left(\mathrm{CDCl}_{3}\right): \delta 8.05(\mathrm{~d}, \mathrm{Ar}-$

$\left.H, 2 \mathrm{H},{ }^{4} J_{\mathrm{HH}} 2.2 \mathrm{~Hz}\right), 7.44\left(\mathrm{~d}, \mathrm{Ar}-H, 2 \mathrm{H},{ }^{4} J_{\mathrm{HH}} 2.2 \mathrm{~Hz}\right), 4.12\left(\mathrm{t},-\mathrm{O}-\mathrm{CH}_{2}-, 4 \mathrm{H},{ }^{3} J_{\mathrm{HH}} 6.6 \mathrm{~Hz}\right), 1.92-1.80$ (m, -O- $\left.\mathrm{CH}_{2}-\mathrm{CH}_{2^{-}}, 4 \mathrm{H}\right), 1.57-1.44\left(\mathrm{~m},-\mathrm{O}-\left(\mathrm{CH}_{2}\right)_{2}-\mathrm{CH}_{2^{-}}, 4 \mathrm{H}\right), 1.43-1.18\left(\mathrm{~m},-\left(\mathrm{CH}_{2}\right)_{6}-\mathrm{CH}_{3}, 24 \mathrm{H}\right), 0.87$ (t, $\left.-\mathrm{CH}_{3}, 6 \mathrm{H},{ }^{3} \mathrm{~J}_{\mathrm{HH}} 7.1 \mathrm{~Hz}\right)$.

Synthesis of 3,6-diethoxycarbonylmethoxy-1,8-naphthalic anhydride. Prepared as described above from 3,6-dihydroxy-1,8-naphthalic anhydride (3.260 g, $14.163 \mathrm{mmol}), \mathrm{NaH}(0.714 \mathrm{~g}, 29.742$ $\mathrm{mmol})$, ethyl bromacetate $(3.5 \mathrm{ml}, 29.742 \mathrm{mmol})$; recrystallization from ethyl acetate. Yield: $2.848 \mathrm{~g}$ 
(50\%); mp $187^{\circ} \mathrm{C}$; MS (FD): m/z $510.8\left(\mathrm{M}^{+}\right) ;{ }^{1} \mathrm{H} \mathrm{NMR}\left(\mathrm{CDCl}_{3}\right): \delta 8.12\left(\mathrm{~d}, \mathrm{Ar}-H, 2 \mathrm{H},{ }^{4} J_{\mathrm{HH}} 1.8 \mathrm{~Hz}\right)$, $7.46\left(\mathrm{~d}, \mathrm{Ar}-\mathrm{H}, 2 \mathrm{H},{ }^{4} \mathrm{~J}_{\mathrm{HH}} 1.8 \mathrm{~Hz}\right), 4.81$ (s, -O-CH 2 -COOEt, $\left.4 \mathrm{H}\right), 4.30$ (q, -O-CH $-\mathrm{CH}_{3}, 4 \mathrm{H},{ }^{3} J_{\mathrm{HH}} 7.0$ $\mathrm{Hz}), 1.31\left(\mathrm{t},-\mathrm{CH}_{3}, 6 \mathrm{H},{ }^{3} \mathrm{~J}_{\mathrm{HH}} 7.0 \mathrm{~Hz}\right)$.

Synthesis of calix[8]arene 4. I (0.050 g, $0.0462 \mathrm{mmol})$, 3,6-dihydroxy-1,8-naphthalic anhydride $(0.106 \mathrm{~g}, 0.462 \mathrm{mmol}), \mathrm{Zn}(\mathrm{OAc})_{2}(0.085 \mathrm{~g}, 0.462 \mathrm{mmol})$. The precipitate was refluxed in acetonitrile $(20 \mathrm{~mL})$ or in methanol $(20 \mathrm{~mL})$, cooled to $\mathrm{rt}$ and filtered off to give compound 4 . Yield $0.112 \mathrm{~g}(87 \%)$; $\mathrm{mp}>370^{\circ} \mathrm{C}$ (decomp.); MS (ESI): m/z $1411.92\left(\mathrm{M}+2 \mathrm{Na}^{+}\right) .{ }^{1} \mathrm{H}$ NMR $\left(\mathrm{DMSO}-\mathrm{d}_{6}\right): \delta 10.24(\mathrm{~s}, \mathrm{Ar}-\mathrm{OH}$, 16 H), 7.66 (s, Ar- H, 16 H), 7.40 (s, Ar-H, 16 H), 6.99 (s, Ar-H, 16 H), 4.08 (s, Ar-CH $-\mathrm{Ar}, 16 \mathrm{H}), 3.46$ (s, $\left.-\mathrm{O}-\mathrm{CH}_{3}, 24 \mathrm{H}\right)$.

Synthesis of calix[8]arene 5. I (0.050 g, $0.0462 \mathrm{mmol})$, 3,6-diethoxycarbonylmethoxy-1,8naphthalic anhydride $(0.186 \mathrm{~g}, 0.462 \mathrm{mmol}),(i-\mathrm{Pr})_{2} \mathrm{NEt}(0.060 \mathrm{~g}, 0.462 \mathrm{mmol})$ has been used instead of $\mathrm{Zn}(\mathrm{OAc})_{2}$ to avoid side reactions. The precipitate was dissolved in dichloromethane $(2-3 \mathrm{~mL})$ and passed through a column (THF/hexane/dichloromethane $(2: 1: 1)$, followed by THF) to collect compound 5. Trituration with hexane $(3 \times 10 \mathrm{~mL})$ finally gave imide 5 as a light-orange powder. Yield $0.034 \mathrm{~g}$ (18\%); $\mathrm{mp}>155^{\circ} \mathrm{C}$ (decomp.); $\mathrm{MS}$ (ESI): m/z $4179.21\left(\mathrm{M}+\mathrm{Na}^{+}\right), 2100.72\left(\mathrm{M}+2 \mathrm{Na}^{+}\right) .{ }^{1} \mathrm{H}$ NMR $\left(\mathrm{CDCl}_{3}\right): \delta 7.92(\mathrm{~s}, \mathrm{Ar}-H, 16 \mathrm{H}), 7.29(\mathrm{~s}, \mathrm{Ar}-H, 16 \mathrm{H}), 6.99(\mathrm{~s}, \mathrm{Ar}-H, 16 \mathrm{H}), 4.72\left(\mathrm{~s},-\mathrm{O}-\mathrm{CH}_{2}-\mathrm{COOEt}\right.$, $32 \mathrm{H}), 4.23\left(\mathrm{q},-\mathrm{O}-\mathrm{CH}_{2}-\mathrm{CH}_{3},{ }^{3} J_{\mathrm{HH}} 7.0 \mathrm{~Hz}, 32 \mathrm{H}\right), 4.13$ (s, Ar-CH$\left.-\mathrm{Ar}, 16 \mathrm{H}\right), 3.57$ (s, -O-CH, $24 \mathrm{H}$ ), $1.25\left(\mathrm{t},-\mathrm{O}-\mathrm{CH}_{2}-\mathrm{CH}_{3},{ }^{3} \mathrm{~J}_{\mathrm{HH}} 7.0 \mathrm{~Hz}, 48 \mathrm{H}\right)$.

Synthesis of calix[8]arene 6. Calix[8] arene I (0.073 g, $0.0675 \mathrm{mmol})$, acetic anhydride $(2 \mathrm{~mL})$, and 2-3 drops of $\mathrm{Et}_{3} \mathrm{~N}$ were refluxed in THF (5 mL) for $5 \mathrm{~min}$ (to dissolve the octa-amine) and stirred for 14 $\mathrm{h}$ at $\mathrm{rt}$. Then THF was removed under reduced pressure and the residue was poured into cold water (25 $\mathrm{mL})$ to form an orange-brown solid. The precipitate was filtered off, washed with water $(2 \times 20 \mathrm{~mL})$ and methanol (2 x $10 \mathrm{~mL})$ and recrystallized from THF (5 mL)/methanol $(10 \mathrm{~mL})$ to give 6 as a lightorange powder. Yield $0.086 \mathrm{~g}(90 \%) ; \mathrm{mp}>240^{\circ} \mathrm{C}$ (decomp.); MS (FD): m/z $1414.4\left(\mathrm{M}^{+}\right) .{ }^{1} \mathrm{H}$ NMR 


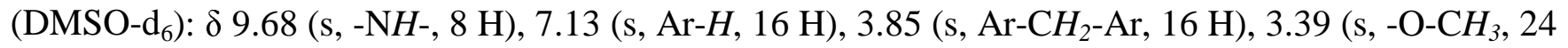
$\mathrm{H}), 1.90\left(\mathrm{~s},-\mathrm{CO}-\mathrm{CH}_{3}, 24 \mathrm{H}\right)$.

Synthesis of calix[8]arene 7. A solution of $p$-toluoyl chloride $(0.033 \mathrm{~g}, 0.213 \mathrm{mmol})$ in THF $(3 \mathrm{~mL})$ was added to a suspension of the calix[8]arene $\mathbf{I}(0.023 \mathrm{~g}, 0.0213 \mathrm{mmol})$ and $\mathrm{Et}_{3} \mathrm{~N}(0.1 \mathrm{~mL}, 0.712$ mmol) in THF (2 mL). The reaction mixture was stirred for $24 \mathrm{~h}$ at $\mathrm{rt}$. Then the THF was removed under reduced pressure and to the residue was triturated with methanol $(10 \mathrm{~mL})$. A thin brown powder was filtered off, and washed with water $(5 \mathrm{~mL})$ and methanol $(2 \times 15 \mathrm{~mL})$. Reprecipitation from THF (2 $\mathrm{mL}) /$ hexane $(10 \mathrm{~mL})$ gave 7 as a thin beige powder. Yield $0.022 \mathrm{~g}(51 \%) ; \mathrm{mp}>235^{\circ} \mathrm{C}$ (decomp.); $\mathrm{MS}$ (FD): m/z $2028.9\left(\mathrm{M}+\mathrm{H}^{+}\right) .{ }^{1} \mathrm{H}$ NMR $\left(\mathrm{DMSO}_{6}\right): \delta 9.98(\mathrm{~s},-\mathrm{NH}-, 8 \mathrm{H}), 7.73\left(\mathrm{~d}, \mathrm{Ar}-H, 16 \mathrm{H},{ }^{3} J_{\mathrm{HH}} 7.7\right.$ Hz), 7.39 (s, Ar- H, 16 H), 7.18 (d, Ar-H, 16 H, $\left.{ }^{3} J_{\mathrm{HH}} 8.1 \mathrm{~Hz}\right), 3.91$ (s, Ar-CH $\left.-\mathrm{Ar}, 16 \mathrm{H}\right), 3.45$ (s, -O$\left.\mathrm{CH}_{3}, 24 \mathrm{H}\right), 2.31\left(\mathrm{~s},-\mathrm{CH}_{3}, 24 \mathrm{H}\right)$.

Synthesis of calix[8]arene 9. A solution of $p$-methoxyphenyl isocyanate $(0.083 \mathrm{~g}, 0.555 \mathrm{mmol})$ in THF (3 mL) was added to a suspension of the calix[8]arene I $(0.050 \mathrm{~g}, 0.0462 \mathrm{mmol})$ in THF (2 mL). The reaction mixture was stirred under nitrogen for $24 \mathrm{~h}$ at $\mathrm{rt}$, the THF was removed under reduced pressure and methanol $(5 \mathrm{~mL})$ was added to the residue to form a brown thin solid that was filtered off, and washed with methanol $(2 \times 10 \mathrm{~mL})$. The light-brown powder was refluxed in THF $(5 \mathrm{~mL}) / \mathrm{methanol}$ $(10 \mathrm{~mL})$ for $5 \mathrm{~min}$, cooled to $\mathrm{rt}$ and filtered off. This operation was repeated twice to give the pure product 9 as a thin light-brown powder. Yield $0.069 \mathrm{~g}(66 \%) ; \mathrm{mp}>500^{\circ} \mathrm{C}$ (decomp.); $\mathrm{MS}(\mathrm{ESI}): \mathrm{m} / \mathrm{z}$ $2297.28\left(\mathrm{M}+\mathrm{Na}^{+}\right), 1160.12\left(\mathrm{M}+2 \mathrm{Na}^{+}\right) .{ }^{1} \mathrm{H}$ NMR $\left(\mathrm{DMSO}_{6}, \mathrm{~d}_{6}, 130^{\circ} \mathrm{C}\right): \delta 8.03(\mathrm{~s},-\mathrm{NH}-, 8 \mathrm{H}), 7.91(\mathrm{~s},-$ $\mathrm{N} H-, 8 \mathrm{H}), 7.23\left(\mathrm{~d}, \mathrm{Ar}-H, 16 \mathrm{H},{ }^{3} J_{\mathrm{HH}} 5.4 \mathrm{~Hz}\right), 7.08(\mathrm{~s}, \mathrm{Ar}-H, 16 \mathrm{H}), 6.78\left(\mathrm{~d}, \mathrm{Ar}-H, 16 \mathrm{H},{ }^{3} J_{\mathrm{HH}} 5.9 \mathrm{~Hz}\right)$, 3.91 (s, Ar- $\mathrm{CH}_{2}$-Ar, $\left.16 \mathrm{H}\right), 3.71$ (s, -O-CH $\left.3,24 \mathrm{H}\right), 3.45$ (s, -O-CH 3 , $24 \mathrm{H}$ ).

Synthesis of calix[8]arene 12. A suspension of the calix[8]arene I $(0.020 \mathrm{~g}, 0.0185 \mathrm{mmol})$ in THF $(5 \mathrm{~mL})$ was added under nitrogen to a stirred solution of 4-(2',3',4',5'-tetraphenyl)biphenyl isocyanate, prepared from the corresponding amine $(0.088 \mathrm{~g}, 0.185 \mathrm{mmol})$, triphosgene $(0,019 \mathrm{~g}, 0.0647 \mathrm{mmol})$ and $(i-\operatorname{Pr})_{2} \mathrm{NEt}(0.010 \mathrm{~g}, 0.0740 \mathrm{mmol})$ in toluene $(100 \mathrm{~mL})$. The reaction mixture was stirred for $48 \mathrm{~h}$ at $\mathrm{rt}$ 
under nitrogen, the solvent was removed under reduced pressure and methanol $(10 \mathrm{~mL})$ was added to form a brown solid which was filtered off and washed with methanol (2 x $10 \mathrm{~mL})$. The powder was dissolved in THF (1 mL) and reprecipitated by hexane $(20 \mathrm{~mL})$ to give 12 as a light-brown powder. Yield $0.049 \mathrm{~g}(52 \%) ; \mathrm{mp}>450^{\circ} \mathrm{C}$ (decomp.); MS (ESI): m/z $2562.35\left(\mathrm{M}+2 \mathrm{Na}^{+}\right) .{ }^{1} \mathrm{H}$ NMR $\left(\mathrm{DMSO}-\mathrm{d}_{6}\right.$, $\left.120^{\circ} \mathrm{C}\right): \delta 8.10(\mathrm{~s},-\mathrm{NH}-, 8 \mathrm{H}), 8.07(\mathrm{~s},-\mathrm{NH}-, 8 \mathrm{H}), 7.39$ (s, Ar-H, $\left.8 \mathrm{H}\right), 6.66-7.28(\mathrm{~m}, \mathrm{Ar}-H, 26 \mathrm{H}), 3.88$ (s, $\left.\mathrm{Ar}-\mathrm{CH}_{2}-\mathrm{Ar}, 16 \mathrm{H}\right), 3.40\left(\mathrm{~s},-\mathrm{O}-\mathrm{CH}_{3}, 24 \mathrm{H}\right)$.

Synthesis of calix[8]arene 15. II $(0.620 \mathrm{~g}, 0.283 \mathrm{mmol})$, 1-ethynylnaphthalene $(0.48 \mathrm{~mL}, 3.375$ $\mathrm{mmol}),\left(\mathrm{Ph}_{3} \mathrm{P}\right)_{2} \mathrm{PdCl}_{2}(0.086 \mathrm{~g}, 0.123 \mathrm{mmol}), \mathrm{Ph}_{3} \mathrm{P}(0.021 \mathrm{~g}, 0.080 \mathrm{mmol}), i-\mathrm{Pr}_{2} \mathrm{NH}(6.0 \mathrm{~mL}, 43 \mathrm{mmol})$, $\mathrm{CuI}(0.013 \mathrm{~g}, 0.068 \mathrm{mmol}), \mathrm{THF}(25 \mathrm{~mL})$. The reaction mixture was filtered and the solid was extracted with $\mathrm{CH}_{2} \mathrm{Cl}_{2}$. The solution was washed with water, dried $\left(\mathrm{MgSO}_{4}\right)$, the solvent was removed in vacuum to give 15 as a pale powder. Yield $0.056 \mathrm{~g}(9 \%) ; \mathrm{mp}>195^{\circ} \mathrm{C}$ (decomp.); $R_{\mathrm{f}}=0.63$ (EtOAc/hexane, 1:1); MS (ESI): m/z $2408.98\left(\mathrm{M}+\mathrm{Na}^{+}\right), 1215.98\left(\mathrm{M}+2 \mathrm{Na}^{+}\right) ;{ }^{1} \mathrm{H} \mathrm{NMR}\left(\mathrm{CDCl}_{3}, 55^{\circ} \mathrm{C}\right): \delta 8.33(\mathrm{~d}, \mathrm{Ar}-H, 8$ $\left.\mathrm{H},{ }^{3} J_{\mathrm{HH}} 7.8 \mathrm{~Hz}\right), 7.77\left(\mathrm{t}, \mathrm{Ar}-H, 16 \mathrm{H},{ }^{3} J_{\mathrm{HH}} 8.0 \mathrm{~Hz}\right), 7.67\left(\mathrm{~d}, \mathrm{Ar}-H, 8 \mathrm{H},{ }^{3} J_{\mathrm{HH}} 7.0 \mathrm{~Hz}\right), 7.39-7.52(\mathrm{~m}, \mathrm{Ar}-$ $H, 16 \mathrm{H}), 7.36\left(\mathrm{~d}, \mathrm{Ar}-H, 8 \mathrm{H},{ }^{3} J_{\mathrm{HH}} 7.4 \mathrm{~Hz}\right), 7.32$ (s, Ar-H, $\left.16 \mathrm{H}\right), 3.71$ (s, Ar-CH $\left.-\mathrm{Ar}, 16 \mathrm{H}\right), 1.98$ (s, $\left.\mathrm{CH}_{3}, 24 \mathrm{H}\right) ;{ }^{13} \mathrm{C} \mathrm{NMR}\left(\mathrm{CDCl}_{3}, 55^{\circ} \mathrm{C}\right): \delta 20.09\left(-\mathrm{CH}_{3}\right), 31.75\left(-\mathrm{CH}_{2^{-}}\right), 88.30(\mathrm{C} \equiv \mathrm{C}), 93.51(\mathrm{C} \equiv \mathrm{C})$, $120.75,121.79,125.21,126.19,126.46,126.91,128.29,128.91,130.53,132.63,133.39,148.08$, $168.23(C=\mathrm{O})$.

Synthesis of N-(4-ethynylphenyl)-1,8-naphthalimide. A mixture of 4-ethynylaniline ( $0.337 \mathrm{~g}, 2.88$ mmol), 1,8-naphthalic anhydride $(0.628 \mathrm{~g}, 3.17 \mathrm{mmol})$, and $\mathrm{Zn}(\mathrm{OAc})_{2}(0.212 \mathrm{~g}, 1.70 \mathrm{mmol})$ in pyridine $(5 \mathrm{~mL})$ was refluxed for $1 \mathrm{~h}$. After cooling to $\mathrm{rt}$ hydrochloric acid (4\% solution, $80 \mathrm{~mL}$ ) was added, a solid was filtered off, washed with water and dried. An attempt to purify the product by column chromatography $\left(\mathrm{CHCl}_{3}\right)$ resulted in a powder $\left(75 \%\right.$ purity as estimated with $\left.{ }^{1} \mathrm{H} \mathrm{NMR}\right)$, which has been used for the next step without further purification. Yield: 0.640 g. ${ }^{1} \mathrm{H} \mathrm{NMR}\left(\mathrm{CDCl}_{3}\right): \delta 8.64(\mathrm{dd}, \mathrm{Ar}-H$, $\left.{ }^{3} J_{\mathrm{HH}} 7.3 \mathrm{~Hz},{ }^{4} J_{\mathrm{HH}} 1.1 \mathrm{~Hz}\right), 8.63\left(\mathrm{dd}, \mathrm{Ar}-H,{ }^{3} J_{\mathrm{HH}} 7.2 \mathrm{~Hz},{ }^{4} J_{\mathrm{HH}} 1.0 \mathrm{~Hz}\right), 8.32\left(\mathrm{~d}, \mathrm{Ar}-H,{ }^{3} J_{\mathrm{HH}} 7.3 \mathrm{~Hz}\right), \delta$ 
$8.23\left(\mathrm{dd}, \mathrm{Ar}-H,{ }^{3} J_{\mathrm{HH}} 7.9 \mathrm{~Hz},{ }^{4} J_{\mathrm{HH}} 1.1 \mathrm{~Hz}\right), 7.71-7.87(\mathrm{~m}, \mathrm{Ar}-H), 7.66\left(\mathrm{~d}, \mathrm{Ar}-H, 2 \mathrm{H},{ }^{3} J_{\mathrm{HH}} 8.5 \mathrm{~Hz}\right), 7.28$

(d, Ar- $\left.H, 2 \mathrm{H},{ }^{3} J_{\mathrm{HH}} 8.5 \mathrm{~Hz}\right), 3.13(\mathrm{~s}, \equiv \mathrm{CH}, 1 \mathrm{H})$.

Synthesis of calix[8]arene 18. II (0.400 g, $0.182 \mathrm{mmol}), \mathrm{N}$-(4-ethynylphenyl)-1,8-naphthalimide (0.640 g, $1.61 \mathrm{mmol}),\left(\mathrm{Ph}_{3} \mathrm{P}\right)_{2} \mathrm{PdCl}_{2}(0.052 \mathrm{~g}, 0.074 \mathrm{mmol}), \mathrm{Ph}_{3} \mathrm{P}(0.016 \mathrm{~g}, 0.061 \mathrm{mmol}), i-\mathrm{Pr}_{2} \mathrm{NH}(4.0$ $\mathrm{mL}, 28.5 \mathrm{mmol})$, CuI (0.013 g, $0.068 \mathrm{mmol})$, THF $(8 \mathrm{~mL})$; reflux, $72 \mathrm{~h}$. The solid was filtered off and washed with THF to give 18 as a powder. Yield: $0.424 \mathrm{~g}(66 \%) ; \mathrm{mp}>320^{\circ} \mathrm{C}$ (decomp.); MS (ESI): $\mathrm{m} / \mathrm{z}$ $1796.58\left(\mathrm{M}+2 \mathrm{Na}^{+}\right) ;{ }^{1} \mathrm{H}$ NMR $\left(\mathrm{DMSO}-\mathrm{d}_{6}, 130^{\circ} \mathrm{C}\right): \delta$ signals for the calixarene skeleton: $7.33(\mathrm{~s}, \mathrm{Ar}-H$, $16 \mathrm{H}), 3.74\left(\mathrm{~s}, \mathrm{Ar}-\mathrm{CH}_{2}-\mathrm{Ar}, 16 \mathrm{H}\right), 2.14\left(\mathrm{~s},-\mathrm{CH}_{3}, 24 \mathrm{H}\right)$; two sets of signals for naphthyl and phenylene units (major (mj) and minor (mn), in 2:1 ratio; the reason of this splitting is not known; the sum of their integral intensities is in accordance with the expected structure): mj: $8.44\left(\mathrm{~d}, \mathrm{Ar}-H,{ }^{3} J_{\mathrm{HH}} 7.1 \mathrm{~Hz}\right), 8.37$ $\left(\mathrm{d}, \mathrm{Ar}-H,{ }^{3} J_{\mathrm{HH}} 7.1 \mathrm{~Hz}\right), 7.79\left(\mathrm{t}, \mathrm{Ar}-H,{ }^{3} J_{\mathrm{HH}} 7.8 \mathrm{~Hz}\right), 7.54$ (d, Ar- $\left.H,{ }^{3} J_{\mathrm{HH}} 8.2 \mathrm{~Hz}\right), 7.30\left(\mathrm{~d}, \mathrm{Ar}-H,{ }^{3} J_{\mathrm{HH}} 8.2\right.$ $\mathrm{Hz}$ ); mn: 8.54 (d, Ar- $\left.H,{ }^{3} J_{\mathrm{HH}} 7.0 \mathrm{~Hz}\right),\left(\mathrm{d}, \mathrm{Ar}-H,{ }^{3} J_{\mathrm{HH}} 7.0 \mathrm{~Hz}\right), 7.90\left(\mathrm{t}, \mathrm{Ar}-H,{ }^{3} J_{\mathrm{HH}} 7.8 \mathrm{~Hz}\right), 7.76(\mathrm{~d}, \mathrm{Ar}-H$, $\left.{ }^{3} J_{\mathrm{HH}} 8.2 \mathrm{~Hz}\right), 7.47\left(\mathrm{~d}, \mathrm{Ar}-H,{ }^{3} J_{\mathrm{HH}} 8.2 \mathrm{~Hz}\right)$.

Synthesis of N-(4-ethynylphenyl)-3,6-dihydroxy-1,8-naphthalimide. Prepared as described above for N-(4-ethynylphenyl)-1,8-naphthalimide from 4-ethynylaniline (0.462 g, 3.60 mmol), 3,6-dihydroxy1,8-naphthalic-anhydride (0.911 g, $3.96 \mathrm{mmol}), \mathrm{Zn}(\mathrm{OAc})_{2}(0.277 \mathrm{~g}, 2.23 \mathrm{mmol})$, and pyridine $(10 \mathrm{~mL})$. After working up with hydrochloric acid (4\% solution, $100 \mathrm{~mL})$ the solid was extracted with $\mathrm{MeOH}$. The solvent was evaporated and the residue was extracted with THF to give 19a as a powder. Yield: $0.719 \mathrm{~g}(61 \%) ; \mathrm{mp}>255^{\circ} \mathrm{C}$ (decomp.); $\mathrm{MS}(\mathrm{FD}): \mathrm{m} / \mathrm{z} 329.3\left(\mathrm{M}^{+}\right) ;{ }^{1} \mathrm{H} \mathrm{NMR}\left(\mathrm{MeOH}-\mathrm{d}_{4}\right): \delta 7.73-7.81$ (m, Ar- H, $4 \mathrm{H}), 7.34$ (d, Ar-H, $\left.2 \mathrm{H},{ }^{4} J_{\mathrm{HH}} 2.2 \mathrm{~Hz}\right), 7.28\left(\mathrm{~d}, \mathrm{Ar}-H, 2 \mathrm{H},{ }^{4} J_{\mathrm{HH}} 2.2 \mathrm{~Hz}\right), 7.61(\mathrm{~d}, \mathrm{Ar}-H, 2 \mathrm{H}$, $\left.{ }^{3} J_{\mathrm{HH}} 8.5 \mathrm{~Hz}\right), 7.32\left(\mathrm{~d}, \mathrm{Ar}-H, 2 \mathrm{H},{ }^{3} J_{\mathrm{HH}} 8.5 \mathrm{~Hz}\right), 3.58(\mathrm{~s}, \equiv \mathrm{CH}, 1 \mathrm{H})$.

Synthesis of N-(4-ethynylphenyl)-3,6-didecyloxy-1,8-naphthalimide. A mixture of N-(4-ethynylphenyl)-3,6-dihydroxy-1,8-naphthalimide (3.71 g, $11.27 \mathrm{mmol}), \mathrm{K}_{2} \mathrm{CO}_{3}(3.94 \mathrm{~g}, 28.51 \mathrm{mmol})$, and decyl bromide $(6.13 \mathrm{~g}, 27.69 \mathrm{mmol})$ in a mixture of acetonitrile $(100 \mathrm{~mL})$ and DMF $(50 \mathrm{~mL})$ was degassed with $\mathrm{N}_{2}$ and refluxed for $5 \mathrm{~h}$. After cooling to $\mathrm{rt}$ the mixture was poured into ice water, the 
product was extracted with chloroform, the organic phase was washed with water, dried $\left(\mathrm{MgSO}_{4}\right)$ and evaporated. Purification by column chromatography $\left(\mathrm{CHCl}_{3} /\right.$ hexane, $\left.3 / 1\right)$ gave the desired product as a powder. Yield: $2.67 \mathrm{~g}(40 \%) ;{ }^{1} \mathrm{H} \mathrm{NMR}\left(\mathrm{CDCl}_{3}\right): \delta 8.08\left(\mathrm{~d}, \mathrm{Ar}-\mathrm{H}, 2 \mathrm{H},{ }^{4} J_{\mathrm{HH}} 2.4 \mathrm{~Hz}\right), 7.42(\mathrm{~d}, \mathrm{Ar}-\mathrm{H}, 2 \mathrm{H}$, $\left.{ }^{4} J_{\mathrm{HH}} 2.4 \mathrm{~Hz}\right), 7.64\left(\mathrm{~d}, \mathrm{Ar}-\mathrm{H}, 2 \mathrm{H},{ }^{3} J_{\mathrm{HH}} 8.2 \mathrm{~Hz}\right), 7.26\left(\mathrm{~d}, \mathrm{Ar}-\mathrm{H}, 2 \mathrm{H},{ }^{3} J_{\mathrm{HH}} 8.2 \mathrm{~Hz}\right), 4.13\left(\mathrm{t},-\mathrm{O}-\mathrm{CH}_{2^{-}}, 4 \mathrm{H}\right.$, $\left.{ }^{3} J_{\mathrm{HH}} 6.5 \mathrm{~Hz},\right), 3.12(\mathrm{~s}, \equiv \mathrm{CH}, 1 \mathrm{H}), 1.79-1.93\left(\mathrm{~m},-\mathrm{O}-\mathrm{CH}_{2}-\mathrm{CH}_{2^{-}}, 4 \mathrm{H}\right), 1.44-1.58\left(\mathrm{~m},-\mathrm{O}-\left(\mathrm{CH}_{2}\right)_{2}-\mathrm{CH}_{2^{-}}, 4\right.$ H), 1.27 (br.s, - $\left.\left(\mathrm{CH}_{2}\right)_{6}-\mathrm{CH}_{3}, 24 \mathrm{H}\right), 0.87\left(\mathrm{t},-\mathrm{CH}_{3}, 6 \mathrm{H},{ }^{3} J_{\mathrm{HH}} 6.9 \mathrm{~Hz}\right)$.

Synthesis of calix[8]arene 19. II (0.409 g, $0.187 \mathrm{mmol}$ ), N-(4-ethynylphenyl)-3,6-didecyloxy-1,8naphthalimide $(1.334 \mathrm{~g}, 2.239 \mathrm{mmol}),\left(\mathrm{Ph}_{3} \mathrm{P}\right)_{2} \mathrm{PdCl}_{2}(0.057 \mathrm{~g}, 0.081 \mathrm{mmol}), \mathrm{Ph}_{3} \mathrm{P}(0.016 \mathrm{~g}, 0.061$ mmol), $i$ - $\mathrm{Pr}_{2} \mathrm{NH}(2.0 \mathrm{~mL}, 14.3 \mathrm{mmol})$, CuI (0.014 g, $\left.0.074 \mathrm{mmol}\right)$, THF (25 mL); reflux, $72 \mathrm{~h}$. After filtration the solvent was removed and the residue was reprecipitated from THF/MeOH. Repeated column chromatography: $\mathrm{CHCl}_{3} /$ hexane (3:1), $\mathrm{CH}_{2} \mathrm{Cl}_{2} /$ hexane $(2: 1)$, and hexane/THF $(3 / 1,1 / 1)$ gave 19 as a powder. Yield: $0.487 \mathrm{~g}(43 \%) ; \mathrm{mp} 170-172^{\circ} \mathrm{C} ; R_{\mathrm{f}}=0.33$ (hexane/THF, 5:1); ${ }^{1} \mathrm{H}$ NMR $\left(\mathrm{CDCl}_{3}\right.$, $\left.55^{\circ} \mathrm{C}\right): \delta 8.05$ (s, Ar- $\left.H, 16 \mathrm{H}\right), 7.36$ (s, Ar- $\left.H, 16 \mathrm{H}\right), 7.55$ (s, Ar-H, 16 H), 7.20 (br.s, Ar- $H, 32 \mathrm{H}$ ), 4.11 (br.s, -O-CH $2_{2}^{-}, 16$ H), 3.73 (br.s, Ar-CH $2_{2}$-Ar, 16 H), 2.07 (br.s, -C(O)- $\mathrm{CH}_{3}, 24 \mathrm{H}$ ), 1.74-1.90 (m, -O$\left.\mathrm{CH}_{2}-\mathrm{CH}_{2^{-}}, 16 \mathrm{H}\right), 1.41-1.55$ (m, -O- $\left.\left(\mathrm{CH}_{2}\right)_{2}-\mathrm{CH}_{2^{-}}, 16 \mathrm{H}\right), 1.27$ (br.s, - $\left.\left(\mathrm{CH}_{2}\right)_{6}-\mathrm{CH}_{3}, 96 \mathrm{H},\right), 0.87$ (t, - $\mathrm{CH}_{3}$, $\left.24 \mathrm{H},{ }^{3} J_{\mathrm{HH}} 6.9 \mathrm{~Hz}\right) .{ }^{13} \mathrm{C} \mathrm{NMR}\left(\mathrm{CDCl}_{3}, 55^{\circ} \mathrm{C}\right): \delta 13.96,20.29,22.62,26.05,29.21,29.26,29.37,29.54$, $31.3,31.87,68.90,89.32,113.40,119.50,119.97121 .41,123.52,124.08,128.94,132.36,135.25$, $135.47,148.16,158.40,163.77,168.40$.

Synthesis of 4-ethynyl-(N-BOC)-aniline. A mixture of 4-ethynylaniline (1.23 g, $10.46 \mathrm{mmol})$ and BOC-anhydride $(3.01 \mathrm{~g}, 13.79 \mathrm{mmol})$ in chloroform $(15 \mathrm{~mL})$ was refluxed for $12 \mathrm{~h}$. After cooling to rt the solvent was evaporated and the residue was crystallized from $\mathrm{CH}_{2} \mathrm{Cl}_{2} /$ hexane to give the desired product as a yellow powder. Yield: $0.954 \mathrm{~g}(42 \%) ; \mathrm{mp} 98^{\circ} \mathrm{C}$; MS (FD): $\mathrm{m} / \mathrm{z} 217.3\left(\mathrm{M}^{+}\right) ;{ }^{1} \mathrm{H}$ NMR $\left(\mathrm{CDCl}_{3}\right): \delta 7.39\left(\mathrm{~d}, \mathrm{Ar}-\mathrm{H}, 2 \mathrm{H},{ }^{3} J_{\mathrm{HH}} 8.5 \mathrm{~Hz}\right), 7.30\left(\mathrm{~d}, \mathrm{Ar}-\mathrm{H}, 2 \mathrm{H},{ }^{3} J_{\mathrm{HH}} 8.5 \mathrm{~Hz}\right) 6.55(\mathrm{~s},-\mathrm{NH}-, 1 \mathrm{H}), 3.00$ $(\mathrm{s}, \equiv \mathrm{CH}, 1 \mathrm{H}), 1.49\left(\mathrm{~s},-\mathrm{CH}_{3}, 9 \mathrm{H}\right)$. 


\section{Synthesis of N-(4-ethynylphenyl)-N'-(3,4,5-tridecyloxyphenyl)urea. A mixture of 3,4,5-}

tridecyloxybenzoic acid $(3.46 \mathrm{~g}, 5.86 \mathrm{mmol})$, diphenyl phosphoryl azide $(1.40 \mathrm{~mL}, 6.50 \mathrm{mmol})$, and $\mathrm{Et}_{3} \mathrm{~N}(2 \mathrm{~mL})$ in benzene $(80 \mathrm{~mL})$ was refluxed for $2 \mathrm{~h}$. After cooling to rt the solvent was evaporated. Then 4-ethynylaniline $(0.503 \mathrm{~g}, 4.32 \mathrm{mmol})$ and THF $(60 \mathrm{~mL})$ were added and the mixture was stirred for $12 \mathrm{~h}$ at $\mathrm{rt}$. The solvent was removed in vacuum, $\mathrm{MeOH}$ was added. A solid was filtered off, washed with $\mathrm{MeOH}$ and dried. Reprecipitation from $\mathrm{CH}_{2} \mathrm{Cl}_{2} / \mathrm{MeOH}$ gave the desired product as a powder. Yield: $2.24 \mathrm{~g}(54 \%) ; \mathrm{mp} 88^{\circ} \mathrm{C}$; MS (FD): m/z $705.5\left(\mathrm{M}^{+}\right) ;{ }^{1} \mathrm{H}$ NMR $\left(\mathrm{CDCl}_{3}\right): \delta 7.40\left(\mathrm{~d}, \mathrm{Ar}-H, 2 \mathrm{H},{ }^{3} J_{\mathrm{HH}}\right.$ $8.5 \mathrm{~Hz}), 7.32\left(\mathrm{~d}, \mathrm{Ar}-H, 2 \mathrm{H},{ }^{3} J_{\mathrm{HH}} 8.5 \mathrm{~Hz}\right), 7.06(\mathrm{~s},-\mathrm{N} H-, 1 \mathrm{H}), 6.71(\mathrm{~s},-\mathrm{N} H-, 1 \mathrm{H}), 6.51(\mathrm{~s}, \mathrm{Ar}-H, 2 \mathrm{H})$, 3.76-4.00 (m, -O-CH $\left.2^{-}, 6 \mathrm{H}\right), 3.01(\mathrm{~s}, \equiv \mathrm{CH}, 1 \mathrm{H}), 1.64-1.82\left(\mathrm{~m},-\mathrm{O}-\mathrm{CH}_{2}-\mathrm{CH}_{2^{-}}, 6 \mathrm{H}\right), 1.10-1.51(\mathrm{~m},-$ $\left.\left(\mathrm{CH}_{2}\right)_{6}-\mathrm{CH}_{3}, 36 \mathrm{H}\right), 0.87\left(\mathrm{t},-\mathrm{CH}_{3}, 3 \mathrm{H},{ }^{3} J_{\mathrm{HH}} 6.6 \mathrm{~Hz}\right)$.

Synthesis of calix[8]arene 21. II $(0.120 \mathrm{~g}, 0.055 \mathrm{mmol}), \quad \mathrm{N}$-(4-ethynylphenyl)-N'-(3,4,5tridecyloxyphenyl)urea $(0.351 \mathrm{~g}, 0.498 \mathrm{mmol}),\left(\mathrm{Ph}_{3} \mathrm{P}\right)_{2} \mathrm{PdCl}_{2}(0.018 \mathrm{~g}, 0.026 \mathrm{mmol}), \mathrm{Ph}_{3} \mathrm{P}(0.008 \mathrm{~g}$, $0.031 \mathrm{mmol}), \mathrm{Et}_{3} \mathrm{~N}(1.0 \mathrm{~mL}, 7.2 \mathrm{mmol}), \mathrm{CuI}(0.001 \mathrm{~g}, 5.3 \mu \mathrm{mol}), \mathrm{THF}(20 \mathrm{~mL})$. The reaction mixture was filtered, the solvent was removed under reduced pressure and the residue was extracted with toluene. After evaporation, the residue was reprecipitated from $\mathrm{THF} / \mathrm{MeOH}$ and further purified by column chromatography $\left(\mathrm{CHCl}_{3} / \mathrm{THF}, 25 / 1\right.$, followed by THF) to give 21 as a powder. Yield: $0.062 \mathrm{~g}$ (18\%); mp > $154^{\circ} \mathrm{C}$ (decomp.); ${ }^{1} \mathrm{H}$ NMR $\left(\mathrm{CDCl}_{3}: \mathrm{DMSO}_{6}, 3: 4, \mathrm{v} / \mathrm{v}, 55^{\circ} \mathrm{C}\right): \delta 8.52(\mathrm{~s},-\mathrm{N} H-, 8 \mathrm{H})$, $8.31(\mathrm{~s},-\mathrm{N} H-, 8 \mathrm{H}), 7.39$ (d, Ar- $\left.H, 16 \mathrm{H},{ }^{3} J_{\mathrm{HH}} 7.8 \mathrm{~Hz}\right), 7.32\left(\mathrm{~d}, \mathrm{Ar}-H, 16 \mathrm{H},{ }^{3} J_{\mathrm{HH}} 7.8 \mathrm{~Hz}\right), 7.14(\mathrm{~s}, \mathrm{Ar}-H$, $16 \mathrm{H}), 6.67(\mathrm{~s}, \mathrm{Ar}-H, 16 \mathrm{H}), 3.89\left(\mathrm{t},-\mathrm{O}-\mathrm{CH}_{2^{-}}, 32 \mathrm{H},{ }^{3} J_{\mathrm{HH}} 6.1 \mathrm{~Hz}\right), 3.80\left(\mathrm{t},-\mathrm{O}-\mathrm{CH}_{2^{-}}, 16 \mathrm{H},{ }^{3} J_{\mathrm{HH}} 6.5 \mathrm{~Hz}\right)$, 3.62 (br.s, Ar- $\mathrm{CH}_{2}$-Ar, $\left.16 \mathrm{H}\right), 1.99$ (br.s, -C(O)-CH3, $24 \mathrm{H}$ ), 1.58-1.80 (m, -O-CH $\left.2^{-} \mathrm{CH}_{2^{-}}, 48 \mathrm{H}\right), 1.03-$ 1.47 (m, $\left.-\left(\mathrm{CH}_{2}\right)_{6}-\mathrm{CH}_{3}, 288 \mathrm{H}\right), 0.84$ (br.s, $\left.-\mathrm{CH}_{3}, 24 \mathrm{H}\right) .{ }^{13} \mathrm{C} \mathrm{NMR}\left(\mathrm{CDCl}_{3}, 55^{\circ} \mathrm{C}\right): \delta 14.05,20.08,22.43$,

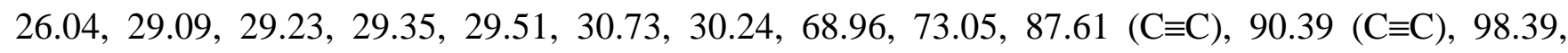
$115.53,118.33,121.34,125.17,132.30,132.76,133.57,135.37,138.81,140.64,147.60,152.64$, $152.97,168.15$. 
Synthesis of N-(4-ethynylphenyl)-3,4,5-tridecyloxybenzamide. A mixture of 3,4,5-tridecyloxy benzoic acid $(3.20 \mathrm{~g}, 5.42 \mathrm{mmol})$ and oxalyl chloride $(4.8 \mathrm{~mL}, 55 \mathrm{mmol})$ in toluene was stirred at $60^{\circ} \mathrm{C}$ for $4 \mathrm{~h}$. Then the solvent and oxalyl chloride were removed under reduced pressure. 4-Ethynylaniline $(0.506 \mathrm{~g}, 4.32 \mathrm{mmol}), \mathrm{Et}_{3} \mathrm{~N}(5 \mathrm{~mL})$ and THF $(10 \mathrm{~mL})$ were added and the mixture was stirred for $12 \mathrm{~h}$ at rt. A solid was filtered off and the solvent was evaporated to give an oil, which was washed with hot $\mathrm{MeOH}$. The remaining oil crystallized upon cooling to give the desired product as a powder. Yield: $1.968 \mathrm{~g}(66 \%) ; \mathrm{mp} \mathrm{58}{ }^{\circ} \mathrm{C} ; \mathrm{MS}(\mathrm{FD}): \mathrm{m} / \mathrm{z} 690.6\left(\mathrm{M}^{+}\right) ;{ }^{1} \mathrm{H} \mathrm{NMR}\left(\mathrm{CDCl}_{3}\right): \delta 7.73(\mathrm{~s},-\mathrm{N} H-, 1 \mathrm{H}), 7.60(\mathrm{~d}$, Ar- $\left.H, 2 \mathrm{H},{ }^{3} J_{\mathrm{HH}} 8.8 \mathrm{~Hz}\right), 7.48\left(\mathrm{~d}, \mathrm{Ar}-H, 2 \mathrm{H},{ }^{3} J_{\mathrm{HH}} 8.8 \mathrm{~Hz}\right), 7.02(\mathrm{~s}, \mathrm{Ar}-H, 2 \mathrm{H}), 3.92-4.12\left(\mathrm{~m},-\mathrm{O}-\mathrm{C} H_{2^{-}}\right.$, $6 \mathrm{H}), 3.05(\mathrm{~s}, \equiv \mathrm{CH}, 1 \mathrm{H}), 1.61-1.90\left(\mathrm{~m},-\mathrm{O}-\mathrm{CH}_{2}-\mathrm{CH}_{2^{-}}, 6 \mathrm{H}\right), 1.10-1.57\left(\mathrm{~m},-\left(\mathrm{CH}_{2}\right)_{7}-\mathrm{CH}_{3}, 48 \mathrm{H}\right), 0.87$ (br.s, $-\mathrm{CH}_{3}, 9 \mathrm{H},{ }^{3} \mathrm{~J}_{\mathrm{HH}} 6.6 \mathrm{~Hz}$ ). 